\title{
FOR EVERY CONTINUOUS $f$ \\ THERE IS AN ABSOLUTELY CONTINUOUS $g$ SUCH THAT $[f=g]$ IS NOT BILATERALLY STRONGLY POROUS

\author{
Z. BUCZOLICH
}

\begin{abstract}
For any Darboux function $f:[0,1] \rightarrow \mathbf{R}$ and any $0<\delta<1$ there is a point $x \in[0,1-\delta]$ and a sequence $x_{n}$ such that

(a) $x_{n} \in\left[x+\delta^{n+1}, x+\delta^{n}\right](n=1,2, \ldots)$ and

(b) $\sum_{n=2}^{\infty}\left|f\left(x_{n}\right)-f\left(x_{n-1}\right)\right|<+\infty$.

Consequently, for every $f \in C[0,1]$ there is an absolutely continuous function $g$ such that $\{x: f(x)=g(x)\}$ is not bilaterally strongly porous.
\end{abstract}

In [1] P. Humke and M. Laczkovich proved that every continuous function agrees with an absolutely continuous function on a set which is not bilaterally strongly $x^{1+\delta}$ porous (Theorem 3 ).

In fact, they proved that for any $f \in C[0,1]$ there is a point $x \in[0,1]$ and a sequence $x_{n} \rightarrow x$ such that

$$
x_{n} \in\left[x+((n+1) !)^{-1-\delta}, x+(n !)^{-1-\delta}\right]
$$

and

$$
\left|f\left(x_{n}\right)-f(x)\right|<n^{-1-\delta / 2}
$$

for $n>n_{0}$ and $\delta>0$.

Let $g \in C[0,1]$ be linear on the intervals $[0, x],\left[x_{n+1}, x_{n}\right](n=1,2, \ldots),\left[x_{1}, 1\right]$, and agree with $f$ at the points $x_{n}$. Since

$$
\sum_{n=n_{0}}^{\infty}\left|f\left(x_{n}\right)-f\left(x_{n-1}\right)\right|<+\infty
$$

$g$ is absolutely continuous and $f=g$ on a set which is not bilaterally strongly $x^{1+\delta}$-porous. We prove the following

THEOREM. For any Darboux function $f:[0,1] \rightarrow \mathbf{R}$ and any $0<\delta<1$ there is a point $x \in[0,1-\delta]$ and a sequence $x_{n}$ such that

(a) $x_{n} \in\left[x+\delta^{n+1}, x+\delta^{n}\right](n=1,2, \ldots)$ and

(b) $\sum_{n=2}^{\infty}\left|f\left(x_{n}\right)-f\left(x_{n-1}\right)\right|<+\infty$.

Defining the function $g$ as above, we obtain the following

COROLlaRY. For every $f \in C[0,1]$ there is an absolutely continuous function $g$ such that $\{x: f(x)=g(x)\}$ is not bilaterally strongly porous.

DEFINITION 1 . Let $R$ be a rectangle $R=[\alpha, \beta] \times[m, M]$. We say that $f$ is in $R$ if graf $\left.f\right|_{[\alpha, \beta]} \subset R$.

Received by the editors January 24, 1986.

1980 Mathematics Subject Classification (1985 Revision). Primary 26A15, 26A46. 
From now on we suppose that we are given a positive number $\delta<1$ and a Darboux function $f:[0,1] \rightarrow \mathbf{R}$.

DEFINITION 2. Suppose that we have a rectangle $R=[\alpha, \beta] \times[m, M]$ and a number $k \in \mathbf{N}$ such that $\delta^{k}<\beta-\alpha$ and $f$ is in $R$. We say that $f$ is $k$-first class in $R$ if whenever $I \subseteq\left[\alpha+\delta^{k+1}, \beta\right]$ with $|I|=\delta^{k}(1-\delta)$, then there exists $x \in I$ such that $f(x)=(M+m) / 2$. Thus we can define a function $y_{k}:\left[\alpha, \beta-\delta^{k}\right] \rightarrow \mathbf{R}$ such that for any $x \in\left[\alpha, \beta-\delta^{k}\right]$ we have $f\left(y_{k}(x)\right)=(m+M) / 2$ and $y_{k}(x) \in\left[x+\delta^{k+1}, x+\delta^{k}\right]$. We say that $f$ is $k$-second class in $R$ if $f$ is not $k$-first class in $R$; that is if there is an $x^{\prime} \in\left[\alpha, \beta-\delta^{k}\right]$ such that $f(x) \neq(m+M) / 2$ for $x \in\left[x^{\prime}+\delta^{k+1}, x^{\prime}+\delta^{k}\right]$.

We choose a number $p \in \mathbf{N}$ such that $\left(1-\delta^{p}\right)-\sum_{n=p}^{\infty} \delta^{n} \geq \delta$.

LEMMA. Suppose that $k \in \mathbf{N} \cup\{0\}, m<M \in \mathbf{R}, \alpha+\delta^{k+1} \in[0,1-\delta], \alpha>0$. For $n \geq k+p$ we define the sequence of rectangles $R_{n}$ by

$$
R_{n}:=\left[\alpha+\delta^{k+1}, \alpha+\delta^{k}\left(1-\delta^{p}\right)-\sum_{l=k+p}^{n-1} \delta^{l}\right] \times[m, M] .
$$

(When $n=k+p$ we define the result of the empty summation to be 0.) We also suppose that $f$ is $n$-first class in $R_{n}$ for $n=k+p, k+p+1, \ldots$ Then there are

$$
x \in\left[\alpha+\delta^{k+1}, \alpha+\delta^{k} \cdot\left(\left(1-\delta^{p}\right)-\frac{\delta^{p}}{1-\delta}\right)\right] \cap[0,1-\delta]
$$

and a sequence $x_{j}$ such that

$$
x_{j} \in\left[x+\delta^{j+1}, x+\delta^{j}\right] \quad(j=1,2, \ldots)
$$

and

$$
\sum_{j=2}^{\infty}\left|f\left(x_{j}\right)-f\left(x_{j-1}\right)\right|<+\infty .
$$

PROOF. By the choice of $p$

$$
I:=\left[\alpha+\delta^{k+1}, \alpha+\delta^{k}\left(\left(1-\delta^{p}\right)-\frac{\delta^{p}}{1-\delta}\right)\right] \neq \varnothing
$$

and we let $x \in I \cap[0,1]$. Since $f$ is $n$-first class in $R_{n}$ we have a function $y_{n}:[\alpha+$ $\left.\delta^{k+1}, \alpha+\delta^{k}\left(1-\delta^{p}\right)-\sum_{l=k+p}^{n} \delta^{l}\right] \rightarrow \mathbf{R}$ and $x$ obviously belongs to the domain of $y_{n}$. We define the desired sequence by $x_{j}:=x+\delta^{j+1}$ for $0<j<k+p$ and $x_{j}:=y_{j}(x)$ for $j \geq k+p$, thus (1) is fulfilled. Since $f\left(y_{j}(x)\right)=(m+M) / 2(j \geq k+p)$ obviously (2) is also fulfilled.

PROOF OF THE THEOREM. First we suppose that in each interval $[\alpha, \beta] \subset$ $[0,1-\delta], \sup \{f(x) ; x \in[\alpha, \beta]\}=+\infty$. Then letting $H_{m}:=\{x \in[0,1] ; f(x) \leq m\}$ we have $\bigcup_{m=1}^{\infty} H_{m}=[0,1]$ and hence, by Baire's theorem, there is an $m_{0}$ and a subinterval $\left[\alpha_{0}, \beta_{0}\right]$ such that $H_{m_{0}}$ is dense in $\left[\alpha_{0}, \beta_{0}\right]$. Thus $f$, as a Darboux function, takes on the value $y=m_{0}+1$ in any subinterval of $\left[\alpha_{0}, \beta_{0}\right]$. Hence the statement of the theorem is obvious.

We can treat similarly the case when $\inf \{f(x) ; x \in[\alpha, \beta]\}=-\infty$ in any subinterval $[\alpha, \beta] \subset[0,1-\delta]$. 
Therefore we can suppose that there is a rectangle $R_{-1}=\left[\alpha_{0}, \beta_{0}\right] \times\left[m_{0}, M_{0}\right]$ such that $f$ is in $R_{-1}$ and $\beta_{0}<1-\delta$. Thus we can choose a number $j_{0}$ such that $\alpha_{0}+\delta^{j_{0}} \leq \beta_{0}$. We put

$$
R_{j_{0}}:=\left[\alpha_{0}+\delta^{j_{0}+1}, \alpha_{0}+\delta^{j_{0}}\left(1-\delta^{p}\right)\right] \times\left[m_{0}, M_{0}\right]
$$

and

$$
\bar{R}_{j_{0}}:=\left[\alpha_{0}+\delta^{j_{0}+1}, \alpha_{0}+\delta^{j_{0}}\right] \times\left[m_{0}, M_{0}\right] .
$$

Obviously $f$ is in $R_{j_{0}}$ and $\bar{R}_{j_{0}}$. By our lemma we can suppose that the conditions of the lemma are not fulfilled by any $k, \alpha \in[0,1-\delta], \beta, m, M$. It follows that there is an index $j_{1} \geq j_{0}+p$ such that

$$
\text { in } R_{j_{1}}^{\prime}:=\left[\alpha_{0}+\delta^{j_{0}+1}, \alpha_{0}+\delta^{j_{0}} \cdot\left(1-\delta^{p}\right)-\sum_{l=j_{0}+p}^{j_{1}-1} \delta^{l}\right] \times\left[m_{0}, M_{0}\right]
$$

$f$ is $j_{1}$-second class

and

$$
\begin{aligned}
& \text { for } j_{0}+p \leq n<j_{1} \text { in } \\
& R_{n}:=\left[\alpha_{0}+\delta^{j_{0}+1}, \alpha_{0}+\delta^{j_{0}}\left(1-\delta^{p}\right)-\sum_{l=j_{0}+p}^{n-1} \delta^{l}\right] \times\left[m_{0}, M_{0}\right]
\end{aligned}
$$

$f$ is $n$-first class.

From (3) it follows that there is

$$
x^{\prime} \in\left[\alpha_{0}+\delta^{j_{0}+1}, \alpha_{0}+\delta^{j_{0}}\left(1-\delta^{p}\right)-\sum_{l=j_{0}+p}^{j_{1}} \delta^{l}\right]
$$

such that $f(x) \neq\left(m_{0}+M_{0}\right) / 2$ for $x \in\left[x^{\prime}+\delta^{j_{1}+1}, x^{\prime}+\delta^{j_{1}}\right]$. We put $\alpha_{1}:=x^{\prime}$.

From the Darboux property of $f$ it follows that we can choose $m_{1}$ and $M_{1}$ such that $M_{1}-m_{1}=\frac{1}{2}\left(M_{0}-m_{0}\right)$ and $f$ is in $\bar{R}_{j_{1}}=\left[\alpha_{1}+\delta^{j_{1}+1}, \alpha_{1}+\delta^{j_{1}}\right] \times\left[m_{1}, M_{1}\right]$ and $\bar{R}_{j_{1}} \subset R_{j_{0}}$. We put

$$
R_{j_{1}}:=\left[\alpha_{1}+\delta^{j_{1}+1}, \alpha_{1}+\delta^{j_{1}}\left(1-\delta^{p}\right)\right] \times\left[m_{1}, M_{1}\right] \quad\left(\subset \bar{R}_{j_{1}} \subset R_{j_{0}}\right) .
$$

Obviously

$$
\left[\alpha_{1}+\delta^{j_{1}+1}, \alpha_{1}+\delta^{j_{1}}\right] \subset\left[\alpha_{0}+\delta^{j_{0}+1}, \alpha_{0}+\delta^{j_{0}}\left(1-\delta^{p}\right)-\sum_{l=j_{0}+p}^{j_{1}-1} \delta^{l}\right] ;
$$

hence, by (4), $x$ belongs to the domain of $y_{n}\left(j_{0}+p \leq n<j_{1}\right)$ for all $x \in\left[\alpha_{1}+\right.$ $\left.\delta^{j_{1}+1}, \alpha_{1}+\delta^{j_{1}}\right]$.

Suppose we have defined $R_{j_{n}}$ for $n \leq i$. Then we repeat our process in $R_{j_{i}}$ and define the index $j_{i+1}$, the numbers $m_{i+1}, M_{i+1}$, the rectangles $R_{j_{i+1}}, \bar{R}_{j_{i+1}}$, and the functions $y_{n}\left(j_{i}+p \leq n<j_{i+1}\right)$. Thus, by induction we can define an infinite sequence of rectangles $R_{j_{i}}, \bar{R}_{j_{i}}(i=0,1, \ldots)$ of numbers $m_{i}, M_{i}(i=0,1, \ldots)$, and we also define the functions $y_{n}$ for $j_{i+1}>n \geq j_{i}+p, i \in \mathbf{N}$. Since $R_{j_{i+1}} \subset R_{j_{i}}$ $(i=0,1, \ldots)$ and $R_{j_{i}}$ is a closed set, $\bigcap_{i=1}^{\infty} R_{j_{i}} \neq \varnothing$; let $(x, y) \in \bigcap_{i=1}^{\infty} R_{j_{i}}$. For 
$0<n<j_{0}+p$ we put $x_{n}:=x+\delta^{n+1}$. If there is an $i \in \mathbf{N}$ such that $j_{i}+p>n \geq j_{i}$ then we also put $x_{n}:=x+\delta^{n+1}$, otherwise we put $x_{n}=y_{n}(x)$. Thus (a) of the theorem is obviously fulfilled. We also have that

$$
\begin{aligned}
\sum_{n=j_{0}+1}^{\infty}\left|f\left(x_{n}\right)-f\left(x_{n-1}\right)\right|= & \sum_{i=0}^{\infty} \sum_{n=j_{i}+p+1}^{j_{i+1}-1}\left|f\left(x_{n}\right)-f\left(x_{n-1}\right)\right| \\
& +\sum_{i=1}^{\infty} \sum_{n=j_{i}}^{j_{i}+p}\left|f\left(x_{n}\right)-f\left(x_{n-1}\right)\right| \\
& +\sum_{n=j_{0}+1}^{j_{0}+p}\left|f\left(x_{n}\right)-f\left(x_{n-1}\right)\right| \\
= & : A_{1}+A_{2}+A_{3} .
\end{aligned}
$$

Since $f\left(x_{n}\right)=\left(M_{i}-m_{i}\right) / 2$ for $j_{i}+p \leq n \leq j_{i+1}-1$, we have $A_{1}=0$. It is easy to check that $\left(x_{l} ; f\left(x_{l}\right)\right) \in \bar{R}_{j_{i-1}}$ for $l \geq j_{i}-1$. Thus $A_{2} \leq \sum_{i=1}^{\infty}(p+1)\left(M_{i-1}-m_{i-1}\right)$. We also have that

$$
M_{i+1}-m_{i+1}=\left(M_{i}-m_{i}\right) / 2 \quad(i=0,1, \ldots) .
$$

Thus $A_{2}<\infty$ and it completes the proof.

\section{REFERENCES}

1. P. Humke and M. Laczkovich, Typical continuous functions are virtually nonmonotone, Proc. Amer. Math. Soc. 94 (1985), 244-248.

Mathematical Institute, Eötvös Loránd University, Budapest, Muzeum KRT. 6-8, H-1088, HUNGARY 